\title{
Anti-Mullerian hormone in young female with different phenotypes of polycystic ovary syndrome without additional risk factors for atherosclerosis in Bulgarian population.
}

\author{
Nikoleta Parahuleva ${ }^{1}$, Ekaterina Uchikova ${ }^{1}$, Anna Mihaylova ${ }^{2}$, Penka Petleshkova ${ }^{\text {* }}$, Mariana S \\ Parahuleva $^{3}$ \\ ${ }^{1}$ Department of Obstetrics and Gynecology, Medical University of Plovdiv, Bulgaria \\ ${ }^{2}$ Medical College, Medical University of Plovdiv, Bulgaria \\ ${ }^{3}$ Internal Medicine Cardiology and Angiology, University Hospital of Giessen and Marburg, Germany
}

\begin{abstract}
Objective: Anti-Mullerian hormone (AMH) was highlighted as a valid marker of ovarian dysfunction in women with anovulatory polycystic ovarian syndrome (PCOS) and was linked with cardiovascular conditions. This study was designed to determine the circulating AMH levels in different phenotypes of PCOS without additional risk factors for atherosclerosis in Bulgarian cohort.

Material and methods: A case-control single-center design was used. The levels of serum AMH were analyzed in non-pregnant, pre-menopausal women with PCOS, smoker or non-smoker, without other risk factors for atherosclerosis.

Results: Significant elevated levels of AMH were observed in all phenotypes of PCOS. However, there are no significant differences between smokers and non-smokers. Serum levels of androgens in PCOS were increased nearly twice. In phenotype $C$ there was a positive correlation between AMH and the androgens. The estradiol levels decreased significantly in the cases with polycystic ovaries. The comparison of the ROC curves shows statistical significant diagnostic efficacy of the variables to diagnose PCOS between AMH and testosteron $(Z=4.586, P<0.001)$ as well as AMH and $E 2(Z=6.450$, $\mathbf{P}<\mathbf{0 . 0 0 1 )}$.

Conclusion: Thus, AMH will be a more reliable diagnostic marker of PCOS than the well-known nonconstant clinical and hormonal parameters in non-pregnant, pre-menopausal women with different phenotypes of PCOS, smokers and non-smokers, without additional risk factors for atherosclerosis in Bulgarian cohort.
\end{abstract}

Keywords: Polycystic ovary syndrome, Anti-Mullerian hormone, Phenotypes, Hormonal parameters, Cardiovascular risk.

Accepted on March 16, 2019

\section{Introduction}

Polycystic Ovarian Syndrome (PCOS) is a common endocrine disorder and cause of anovulatory infertility, affecting $10 \%$ to $15 \%$ of women of reproductive age [1]. PCOS presents itself with a wide spectrum of manifestations which may also include obesity, hyperlipidemia, insulin resistance, diabetes mellitus type II and possibly cardiovascular disease [2,3]. In various parts of the world, PCOS is currently being diagnosed on the basis of Rotterdam criteria which diagnosis the syndrome on the presence of at least two of the following three features: Hyperandrogenism (HA), Oligomenorrhea (OA), and Polycystic ovaries (PCO) [4]. According to this definition, there are four different phenotypes in PCOS: phenotype A (HA $+\mathrm{OA}+\mathrm{PCO})$; phenotype $\mathrm{B}(\mathrm{HA}+\mathrm{OA})$, phenotype $\mathrm{C}$ (HA $+\mathrm{PCO})$, and phenotype $\mathrm{D}(\mathrm{OA}+\mathrm{PCO})[3,4]$. In recent years, it has been reported that, in women with PCOS, circulating antiMullerian hormone (AMH) levels are elevated up to 2-3 times, reflecting the load of growing follicles [5]. In the light of current literature, AMH was highlighted as a valid marker of ovarian ageing and correlates specifically with the other clinico-laboratory parameters of PCOS [6-8]. The recent studies have linked circulating AMH levels with cardiovascular conditions and with the susceptibility to experimentally induced atherosclerosis in rhesus monkey females $[6,9,10]$. It seems that $\mathrm{AMH}$ will soon secure a place in Rotterdam criteria as a diagnostic marker for PCOS [6]. Currently there are no studies in Bulgaria focusing on the association between the phenotype of PCOS patients and circulating AMH levels, as well as potential risk of cardiovascular disease. Therefore, the main focus of the 
present study was to determine the circulating levels of $\mathrm{AMH}$ in young, non-pregnant, pre-menopausal Bulgarian women, smokers and non-smokers, with different phenotypes of PCOS and normo ovulatory patients without additional risk factors for atherosclerosis. Furthermore, we compared circulating levels of AMH and other clinico-laboratory parameters to determine the relationship between $\mathrm{AMH}$ and other hormonal markers as well as its diagnostic efficacy in Bulgarian women with PCOS.

\section{Materials and Methods}

\section{Study population}

We performed a case-control analysis among non-pregnant, pre-menopausal bulgarian women with established diagnosis of PCOS, smokers and non-smokers, aged 18-35 (mean age $22 \pm$ 4.3 years) with no history of cardiovascular disease and without additional risk factors for atherosclerosis $(n=120$, Table 1). The diagnosis of PCOS was made according to conventional Rotterdam criteria 82-4). The participants were averaged in four groups according to four different phenotypes 83-4). Menstrual cycles were recorded for $\geq 3$ months and oligomenorrhea was defined as irregular menstrual cycles at intervals of at least $>35$ days. The smokers were defined as women who smoked 10 or more cigarettes per day. For controls, we selected a group of healthy women who remained free of reported cardiovascular disease and are age and BMImatched with the group of women with PCOS. A control group had no history of pregnancy or recent diseases and were equivalent for blood pressure and the other traditional cardiovascular risk factors included lipid profile and serum glucose $(n=99$, Table 1). Other causes for hyperandrogenism that mimic PCOS such as congenital adrenal hyperplasia, Cushing syndrome, or androgen secreting tumors were excluded from this study. All women were free of infection and did not use any other medication at the time of blood collection. The study was approved by the local ethic council (date of issue January 2013 with registration number 11/2013) and all subjects gave their informed consent to participate in the study.

\section{Laboratory analyses}

All subjects underwent a complete history and physical examination, biochemical analyses and ultrasonography. To obtain serum probes, blood was centrifuged at 3,000g for 20 min at room temperature, and the serum was frozen in aliquots and stored at $-70^{\circ} \mathrm{C}$ until analysis. Routine blood chemistry, lipids and serum glucose were analyzed using fresh blood samples according to established enzymatic methods at the local laboratories. The circulating levels of $\mathrm{LH}, \mathrm{FSH}$, testosterone (T), androstendion (A), estradiol (E2) and AMH were measured during the early follicular phase (day 3-5) of spontaneous menstrual cycle or progestin-induced uterine bleeding in oligo/ amenorrhea women. Serum AMH was determined by ELISA, according to manufacturer's instructions and expressed in $\mathrm{ng} / \mathrm{ml}$.

\section{Transvaginal sonography}

In all patients and control subjects ovarian morphology was assessed using a transducer frequency of $8-10 \mathrm{MHz}$ (GE Voluson 730, Simens, Germany) so that the number of small follicles $(<10 \mathrm{~mm})$ could be found. Ovarian morphology was determined, as was the presence, size, and total number of ovarian follicles.

\section{Statistical analysis}

Data are presented as mean \pm standard deviation (SD). The Mann-Whitney U-test was used to evaluate the differences of circulating hormones between the groups. As the results were not normally distributed, non-parametric Kruskal-Wallis test was used. Correlations were carried out using the Pearson method. ROC analysis was performed to assess the diagnostic efficacy of the variables. A P value of 0.05 or less was considered statistically significant. Statistical analyses were carried out using SPSS 22.0 (SPSS Inc., Chicago, IL, USA).

\section{Results}

The clinical characteristics of the study population are listed in Table 1. There were no significant differences between PCOS group and the control group in the following categories: age, incidence of adipositas, concentrations of total cholesterol, HDL cholesterol, LDL cholesterol, triglycerides, and serum glucose. The percentages of active smokers were similar in all groups

Table 1. Baseline characteristics of study participants.

\begin{tabular}{llll}
\hline Parameters & PCos $\mathbf{n = 1 2 0}$ & Control $\mathbf{n = 9 9}$ & p-value \\
\hline Age (years) & $22 \pm 4.3$ & $24 \pm 5.6$ & n.s. \\
\hline BMl, kg/m2 & $27.21 \pm 0.79$ & $26.7 \pm 2.18$ & n.s. \\
\hline Systolic BP, $\mathrm{mmHg}$ & $117 \pm 8$ & $110 \pm 5$ & n.s. \\
\hline Diastolic BP, $\mathrm{mmHg}$ & $75 \pm 8$ & $75 \pm 10$ & n.s. \\
\hline Cholestrol, mmo/L & $5.90 \pm 1.0$ & $5.90 \pm 0.7$ & n.s. \\
\hline Triglycerides, mmol/L & $1.45 \pm 0.6$ & $1.50 \pm 0.8$ & n.s. \\
\hline LDL cholesterol, mmo/L & $3.4 \pm 0.6$ & $3.3 \pm 0.8$ & n.s. \\
\hline HDL cholesterol, mmo/L & $1.4 \pm 0.6$ & $1.3 \pm 0.5$ & n.s. \\
\hline Serum glucose, mmol/l & $4.634 \pm 0.16$ & $4.5890 \pm 0.15$ & n.s. \\
\hline Smokers, \% & 54 & 50 & n.s. \\
\hline
\end{tabular}

BMI: Body Mass Index; BP: Blood Pressure; LDL: Low Density Lipoprotein HDL: High-Density-Lipoprotein. Values are mean \pm SD. Data were compared and $p$-values are calculated by Kruskal-Wallis test and indicate the difference between a control group and patients group. ns (not significantly) versus control group.

In this prospective, case-control study we obtained 120 young women diagnosed with PCOS based on Rotterdam criteria without any additional risk factors for atherosclerosis and 99 matched controls. Comparison of the mean values of the 

risk factors for atherosclerosis in Bulgarian population

analyzed variables in women with PCOS and in healthy women is presented in Table 2 .

Table 2. Comparative analysis of the investigated parameters in the $P C O S$ and control group.

\begin{tabular}{llll}
\hline Parameters & PCOS $\mathbf{n = 1 2 0}$ & Control $\mathbf{n = 9 9}$ & p-value \\
\hline AMH ng/ml & 15.126 & 3.753 & $* * *$ \\
\hline FSH mlU/mL & 5.185 & 6.047 & n.s. \\
\hline LH IU/mL & 10.725 & 5.218 & $* *$ \\
\hline Testosterone $\mathrm{ng} / \mathrm{ml}$ & 0.789 & 0.431 & $*$ \\
\hline Androstenedione ng/ml & 2.794 & 1.964 & $*$ \\
\hline Estradiol pmol/ L & 198.458 & 318.37 & $* *$ \\
\hline LH/FSH & 2.154 & 0.894 & $*$ \\
\hline
\end{tabular}

AMH: Anti-Mullerian Hormone; FSH: Follicle Stimulating Hormone; LH Luteinising Hormone. Samples were analyzed in triplicates. ${ }^{*} \mathrm{p}<0.05,{ }^{* *} \mathrm{p}<0.01$ ${ }^{* * *} p<0.01$, ns (not significantly) compared to age- and gender-matched controls.

A significant increase in $\mathrm{AMH}$ levels was observed in the group with PCOS compared to the control group (Table 2, $\mathrm{p}<0.001)$. Serum levels of androgens in PCOS were increased nearly twice $(p<0.05)$. The estradiol (E2) levels decreased significantly in the cases with polycystic ovaries compare to healthy women $(\mathrm{p}<0.01)$. Mean value of $\mathrm{LH}$ in the group with PCOS was significantly higher compare to the controls $(\mathrm{p}<0.01)$. LH/FSH ratio were also significantly higher in the group with PCOS compare to the control group $(\mathrm{p}<0.05)$.

\section{Phenotypic correlations in investigated clinicolaboratory parameters}

The highest $\mathrm{AMH}$ values were observed in phenotype $\mathrm{C}$ ( $p=0.541$, Table 3 ). The lowest values of E2, FSH as well as androgens were observed in phenotype $\mathrm{C}$ and $\mathrm{D}$, respectively, compare to the other phenotypes (Table 3). This particular hormonal constellation resulted in a distinct intraovarian hyperandrogenism despite the lowest serum androgen levels in phenotype $\mathrm{C}$, compare to the other phenotypes. In phenotype $\mathrm{A}$ (classical phenotype of PCOS) the changes in the hormonal levels were similar to the established mean values in all patients with PCOS (Table 3).

The mean values of AMH and E2 in this phenotype group were completely comparable with the mean values of the two hormones in all the examined patients with PCOS (Table 3). However, there were no significant differences in AMH levels as well as in the level of the other hormones between smokers and non-smokers (Table 3).

Table 3. Phenotypic distribution of the mean values of the investigated parameters.

\begin{tabular}{|c|c|c|c|c|c|c|c|c|c|}
\hline \multirow{2}{*}{ Parmeters } & \multicolumn{2}{|c|}{ Phenotype A $(n=30)$} & \multicolumn{2}{|c|}{ Phenotype B (n=30) } & \multicolumn{2}{|c|}{ Phenotype C $(n=30)$} & \multicolumn{2}{|c|}{ Phenotype D $(n=30)$} & \multirow[t]{2}{*}{ p-value } \\
\hline & Smokers & Non-smokers & Smokers & Non-smokers & Smokers & Non-smokers & Smokers & Non-smokers & \\
\hline AMH ng/ml & $14.99 \pm 1.1$ & $15.23 \pm 0.9$ & $12.78 \pm 1.2$ & $13.51 \pm 1.0$ & $15.88 \pm 1.1$ & $16.03 \pm 0.7$ & $14.88 \pm 1.7$ & $15.30 \pm 0.1$ & n.s. \\
\hline FSH mIU/MI & $5.32 \pm 0.11$ & $5.22 \pm 0.13$ & $4.98 \pm 0.22$ & $5.19 \pm 0.18$ & $5.5 \pm 0.02$ & $5.20 \pm 0.05$ & $5.05 \pm 0.1$ & $4.95 \pm 0.15$ & n.s. \\
\hline LH IU/mL & $11.23 \pm 0.3$ & $10.43 \pm 0.7$ & $9.97 \pm 0.8$ & $10.11 \pm 0.5$ & $9.87 \pm 0.55$ & $10.57 \pm 0.15$ & $11 \pm 0.2$ & $10.96 \pm 0.5$ & n.s. \\
\hline Testosterone $\mathrm{ng} / \mathrm{ml}$ & $0.88 \pm 0.07$ & $0.95 \pm 0.04$ & $0.83 \pm 0.1$ & $0.91 \pm 0.07$ & $0.39 \pm 0.05$ & $0.43 \pm 0.01$ & $0.94 \pm 0.06$ & $1,23 \pm 0.01$ & $* * *$ \\
\hline Androstenedione $\mathrm{ng} / \mathrm{ml}$ & $3.15 \pm 0.09$ & $3.05 \pm 0.11$ & $2.69 \pm 0.14$ & $3.03 \pm 0.09$ & $1.75 \pm 0.15$ & $2.05 \pm 0.10$ & $3.20 \pm 0.08$ & $3.5 \pm 0.06$ & $\star \star *$ \\
\hline Estradiol pmol/l & $188.2 \pm 18.5$ & $190.7 \pm 17.7$ & $209.5 \pm 25.3$ & $211.9 \pm 20.1$ & $187.3 \pm 17.3$ & $190.9 \pm 19.3$ & $190.2 \pm 17.9$ & $187.2 \pm 18.9$ & n.s. \\
\hline
\end{tabular}

AMH: Anti-Mullerian Hormone; FSH: Follicle Stimulating Hormone; $\mathrm{LH}$ : Luteinising Hormone. Values are mean $\pm \mathrm{SD}$. Data were compared and $\mathrm{p}$ values are calculated by Kruskall-Wallis test and indicate the difference between all groups. ${ }^{* * *} \mathrm{p}<0.001$ and $\mathrm{ns}$ (not significantly) versus phenotype $\mathrm{C}$.

There was a marked negative correlation between AMH and FSH in the groups with phenotype A $(r=-0.754, p<0.01)$ and $B$ $(\mathrm{r}=-0.674, \mathrm{p}<0.01)$, which resulting in oligoanovulation. In the phenotype A there was a strong positive correlation between $\mathrm{AMH}$ and $\mathrm{LH} / \mathrm{FSH}$ ratio $(\mathrm{r}=0.864, \mathrm{p}<0.01)$ as well as $\mathrm{LH}$ $(\mathrm{r}=0.747, \mathrm{p}<0.01)$, which is responsible for the high intraovarian androgenemia. There was a significant inverse correlation between AMH and E2 $(r=-0.588, p<0.01)$ for the whole group with PCOS. In phenotypes A and B there was a much stronger correlation between the high levels of $\mathrm{AMH}$ and the low levels of E2 (rphenotype $A=-0.916, p<0.01$, rphenotype $\mathrm{B}=-0.879, \mathrm{p}<0.01$, respectively). In the group of phenotype $\mathrm{D}$ we found a straight correlation between $\mathrm{AMH}$ and $\mathrm{LH}(\mathrm{r}=0.485, \mathrm{p}<0.01)$ as well as $\mathrm{AMH}$ and $\mathrm{LH} / \mathrm{FSH}$ ratio $(\mathrm{r}=0.185, \mathrm{p}=0.056)$. In this group there was no oligo/ anovulation and no correlation between $\mathrm{AMH}$ and $\mathrm{FSH}$ $(\mathrm{r}=-0.254, \mathrm{p}=0.074)$. However, in phenotype $\mathrm{D}$ there was a strong inverse correlation between $\mathrm{AMH}$ and $\mathrm{E} 2 \mathrm{r}=-0.605$, $\mathrm{p}<0.01$ ). In phenotype $\mathrm{C}$, the group without hyperandrogenism, there was a straight correlation between AMH and the serum levels of androgens $(\mathrm{r}=0.403, \mathrm{p}<0.05)$. The measured value of testosterone in this group was close to the reference values (Table 3). There was no correlation between AMH and the following parameters: E2, LH, FSH, LH/FSH ratio and serum glucose in phenotype $\mathrm{C}$ group.

To better characterize the joint association between all investigated parameters/ hormones and their clinical efficacy to 
diagnose PCOS, we constructed ROC that includes the interaction of $\mathrm{AMH}$ and serum levels of $\mathrm{E} 2, \mathrm{LH}$, testosterone in both smokers and non-smokers. The comparison of the ROC curves shows statistical significant diagnostic efficacy of the variables to diagnose PCOS as follows: between AUC of AMH and $\mathrm{LH}(\mathrm{Z}=2.985, \mathrm{p}<0.001)$; between $\mathrm{AMH}$ and testosteron $(\mathrm{Z}=4.586, \mathrm{P}<0.001)$; $\mathrm{AMH}$ and $\mathrm{E} 2(\mathrm{Z}=6.450, \mathrm{P}<0.001)$.

\section{Discussion}

The recent studies was highlighted the circulating $\mathrm{AMH}$ as a valid marker of ovarian ageing and have linked it with cardiovascular conditions [2,3]. In this case-control study of non-pregnant, pre-menopausal Bulgarian women with established diagnosis of PCOS with no history of cardiovascular disease and without additional risk factors for atherosclerosis we observed a strong trend towards high circulation levels of AMH. In PCOS we found no proportionality in the increase of $\mathrm{AMH}$ and androgens despite their high serum levels.

A positive correlation between $\mathrm{AMH}$, testosteron and androstenedione, was found in healthy women and in the normoandrogenic patients with phenotype C. However, the higher level of LH in women with phenotype $\mathrm{C}$, stimulating androgen synthesis, leads to a marked intraovarian hypeandrogenism despite the lowest serum androgen levels and highest level of AMH.

Furthermore, in the context of hypoestrogenemia with the low values of FSH and E2 there was an unfavorable proportional interaction between the hormones. We may conclude that according to our results androgens demonstrated a proportional positive correlation with AMH when their values were close to the physiological levels. This was also demonstrated in the group with healthy women, where androgens were within reference range, the correlation coefficients were: $\mathrm{rAMH}$ and Testoterone $=0.208, \quad \mathrm{p}=0.039 ; \quad \mathrm{rAMH} \quad$ and androstenedione $=0.265, \mathrm{p}=0.008$, respectively.

In hyperandrogenic types A, B and D we found no significant correlation between AMH and androgens. Thus, in the present study we confirmed the arguable correlations between androgens and $\mathrm{AMH}$, reported by other researchers $[3,4]$. Circulating $\mathrm{AMH}$ decreases in transsexual women becoming male after therapy with testosterone $[11,12]$. Thus, an artificial increase in the serum level of testosterone may decrease circulating $\mathrm{AMH}$, instead of increasing it as expected [12].

However, ultrasound is not a reliable diagnostic method in PCOS in adolescence and in overweight women, in contrast to $\mathrm{AMH}$ which is easily measurable. The high level of $\mathrm{AMH}$ is a constant diagnostic criterion in all four phenotypes and thus it is more reliable for diagnosing PCOS than the traditional nonconstant criteria.

The correlation coefficients between $\mathrm{AMH}$ and the other parameters in the different phenotypes of PCOS demonstrated some specific characteristics of the hormonal interactions. Our results showed that $\mathrm{AMH}$ was a better diagnostic marker than testosteron from practical point of view, as AMH was increased in all phenotypes in contrast to testosteron. An important finding in the study is that we confirmed a statistically significant higher diagnostic reliability of $\mathrm{AMH}$ compare to testosteron to diagnose PCOS. However, contrary to our expectations, there were no significant differences between smokers and non-smokers in AMH levels [13].

\section{Conclusion}

We conclude that smoking do not alter the AMH circulating levels and therefore $\mathrm{AMH}$ could be used as a reliable alternative diagnostic criterion in young women with PCOS, smokers and non-smokers, without any additional risk factors for atherosclerosis. Thus, it is possible that with growing age and cigarette consumption in smokers the progressive $\mathrm{AMH}$ up-regulation takes place. The results in the combination of other cardiovascular risk factors could be differing from the expected outcome and that must be confirmed in larger randomized studies.

\section{References}

1. Norman RJ, Dewailly D, Legro RS, Hickey TE. Polycystic ovary syndrome. Lancet 2007; 370:685-697.

2. ESHRE/ASRM ESHRE/ASRM Rotterdam consensus meeting revised 2003 consensus on diagnostic criteria and long-term health risks related to polycystic ovarysyndrome (PCOS) Hum Reprod 2004; 19:41-47.

3. Fruzzetti F, Perini D, Lazzarini V, Parrini D, Genazzani AR. Adolescent girls with polycystic ovary syndrome showing different phenotypes have a different metabolic profile associated with increasing androgen levels. Fertil Steril. 2009; 92:626-634.

4. Lauritsen MP, Bentzen JG, Pinborg A, Loft A, Forman JL, Thuesen LL, Cohen A, Hougaard DM, Nyboe Andersen A. The prevalence of polycystic ovary syndrome in a normal population according to the Rotterdam criteria versus revised criteria including anti-Mullerian hormone. Hum Reprod 2014; 29:791-801.

5. Bungum L, Franssohn F, Bungum M, Humaidan $\mathrm{P}$, Giwercman A. The circadian variation in anti-Mullerian hormone in patients with polycystic ovary syndrome differs significantly from normally ovulating women. PLoS One 2013; 8:e68223.

6. Pigny P, Merlen E, Robert Y Cortet-Rudelli C, Decanter C, Jonard S, Dewailly D. Elevated serum level of antimullerian hormone in patients with polycystic ovary syndrome: relationship to the ovarian follicle excess and to the follicular arrest. J Clin Endocrinol Metab 2003; 88:5957-5962.

7. Bako AU, Morad S, Atiomo WA. Polycystic ovary syndrome: an overview. Rev Gynecol Pract 2005; 5:115-122.

8. Eilertsen TB, Vanky E, Carlsen SM. Anti-Mullerian hormone in the diagnosis of polycystic ovary syndrome: 
can morphologic description be replaced? Hum Reprod 2012; 27:2494-2502.

9. Dennis NA, Houghton LA, Jones GT, Van Rij AM, Morgan K, McLennan IS. The level of serum antiMullerian hormone correlates with Vitamin D status in men and women but not in boys. J Clin Endocrinol Metab 2012; 97:2450-2455.

10. Appt SE, Chen H, Clarkson TB, Kaplan JR. Premenopausal antimullerian hormone concentration is associated with subsequent atherosclerosis. Menopause 2012; 19:1353-1359.

11. Crisosto N, Sir-Petermann T, Greiner M, Maliqueo M, Moreno M, Aedo P, Lara HE. Testosterone-induced downregulation of anti-Mullerian hormone expression in granulosa cells from small bovine follicles. Endocrine 2009; 36:339-345.

12. Caanen M, Soleman R, Kuijper E, Kreukels BP, De Roo C, Tilleman K, De Sutter P, Van Trotsenburg MA, Broekmans FJ, Lambalk CB. Anti-mullerian hormone serum levels decrease in female to male transsexual women using testosterone as cross-sex therapy. Fertil Steril 2015; 103:1340-1350.
13. Mosca L, Benjamin EJ, Berra K, Bezanson JL, Dolor RJ, Lloyd-Jones DM, Newby LK, Piña IL, Roger VL, Shaw LJ, Zhao D, Beckie TM, Bushnell C, D'Armiento J, KrisEtherton PM, Fang J, Ganiats TG, Gomes AS, Gracia CR, Haan CK, Jackson EA, Judelson DR, Kelepouris E, Lavie CJ, Moore A, Nussmeier NA, Ofili E, Oparil S, Ouyang P, Pinn VW, Sherif K, Smith SC Jr, Sopko G, ChandraStrobos N, Urbina EM, Vaccarino V, Wenger NK American Heart Association. Effectiveness-based guidelines for the prevention of cardiovascular disease in women-2011 update: A guideline from the American Heart Association. J Am Coll Cardiol 2011; 57:1404-1423.

\section{*Correspondence to}

Dr. Penka Petleshkova

Department of Obstetrics and Gynecology

Medical University of Plovdiv

Bulgaria 